\title{
Determinants of unusual and differential longevity: an introduction
}

\author{
Graziella Caselli and Marc Luy*
}

\section{Introduction}

The 2013 issue of the Vienna Yearbook of Population Research presents a series of studies on the determinants of unusual and differential longevity. In the Western world, life expectancy has increased continuously during the last 150 years, leading some experts to speak of 'broken limits to life expectancy' (Oeppen and Vaupel 2002). We are witnessing a real revolution, made possible by major reductions in mortality among elderly persons. Today, more than half the population in the Western world survive until their 80th birthday. The number of people between 90 and 100 years is gradually increasing and the age at death is continuously pushed forward to old and oldest-old ages (Wilmoth et al. 2000). Moreover, the number of those dying after age 100 is growing at a higher rate of increase than that for preceding age groups (Caselli et al. 2006a; Robine et al. 2006a, 2006b).

The unexpected deceleration of the increase in the force of mortality among the oldest-old has called into question the well-known Gompertz's Law (1825) of human longevity, encouraging a wide-ranging debate on the reasons for the extension of the human lifespan (Finch and Pike 1996). Biologists and geneticists who study the mechanisms entailed and try to establish at what stage a person is old developed numerous theories on ageing. According to Medvedev (1990), these can be grouped in four main categories: programmed genetic ageing, age-associated changes, primary damage and evolutionary theories. Some of these theories focus on 'how we age', others on 'why we age' (Williams 1957; Kirkwood 1990; Kirkwood and Rose 1991; Finch and Kirkwood 2000).

In the new millennium the number of centenarians has increased to such an extent as to capture the interest of academics from various disciplines to search for the causes of longevity. The first data on the possible determinants of extreme longevity emerged from two major hypotheses, the first regarding a general conceptualisation of the possible biological determinants of the ageing process, and the second originating from studies on the immunological status of centenarians (Capri et al. 2008). The

\footnotetext{
* Marc Luy (correspondence author), Wittgenstein Centre for Demography and Global Human Capital (IIASA, VID/ÖAW, WU), Vienna Institute of Demography of the Austrian Academy of Sciences, Austria. Email: marc.luy@oeaw.ac.at

Graziella Caselli, Department of Statistical Sciences, Sapienza University of Rome, Italy.
} 
first hypothesis (Kirkwood and Franceschi 1992) is based on the fact that the ageing process is indirectly controlled by a network of cellular and molecular defence mechanisms, including antioxidant defence, heat shock proteins and DNA repair mechanisms. The second hypothesis is based on the assumption that the ageing of the immune system plays a central role in the ageing process (Franceschi et al. 1995). Many studies carried out on centenarians - pioneer studies of the genetic approach to the longevity trait that started from a series of analyses (Pes et al. 2004; Franceschi et al. 2005) - focused on apolipoproteins, a topic that was thoroughly analysed subsequently, including studies on specific genes (such as the well-known APOE). The extensive literature on the topic was presented in a recent work by Capri et al. (2008).

In contrast to the bio-molecular studies, demographers are interested in 'when we die'. Demographic research is focusing primarily on the reasons why some people die at age 60, others at 80 and a few at 100, and why the number of people dying at older ages is increasing so rapidly (Caselli et al. 2006b). These research approaches are important because the current bio-genetic theories of 'how we age' and 'why we age' are too abstract and too general to provide deep insights into the age patterns of mortality and changes in these patterns over time.

Demographers, with few if any theories, have focused on empirical changes in mortality over age and time, considering the fact that the mortality curve is intrinsically statistical. This can provide structural information about a population's extinction, even if none of the individual members behaves like the population as a whole. An interesting fact is that a deceleration of the survival curve does not imply that the speed of individual ageing changes with age. One explanation may be that individuals in the same generation are vulnerable in different ways to death: there are frailer individuals who age faster and die earlier; and there are more robust individuals who age more slowly and live longer. Thus, the observed deceleration in the force of mortality may be caused by a change in the composition of the population when fewer and fewer frail individuals but more and more robust ones are still alive at older ages (Vaupel et al. 1979). This holds even when the mortality age pattern of both frail and robust individuals follows Gompertz's Law.

Considering the effects of this observed or unobserved heterogeneity, demographers have been speculating about the idea of an age limit for the human lifespan. Some experts have suggested that there is no sign of convergence toward a limit, demonstrating that the effect of selection may allow the strongest/healthiest people who have reached retirement age to survive until 100, and some of them could theoretically even reach the ages 115-117 (Barbi et al. 2003). That Jeanne Calment, the most studied and most authenticated super-centenarian in the world, died at the age of 122 and a half supports the hypothesis that there is no fixed limit to human lifespan.

Historically, some bio-demographers referred to the shape of the survival curves in studying the limit of human longevity (Pearl and Doering 1923; Deevey 1947). The notion of 'rectangularisation' of the survival curve was introduced by Comfort $(1956,1964)$ and later advanced by Fries $(1980)$. The latter argued that the human 
survival curve tends to become rectangular because an upper boundary to human life expectancy at 85 years is determined by fixed genetic limits (Fries 1980, 1984; see also Hayflick 1981). The discussions about the links between the rectangularisation of the survival curve and the limits to human life became more intensive in recent years (Olshansky et al. 2001). Wilmoth and Horiuchi (1999) argued that the human survival curve can never be perfectly rectangular in the sense of zero variability in age at death, and Wilmoth (2001) as well as Oeppen and Vaupel (2002) argued that the belief in life expectancy approaching a ceiling has been proven wrong. Note that life expectancy of Japanese women today is about 87 years and their modal age at death is higher than 90 years.

Today there is no general consensus about the definition of rectangularisation of the survival curve and how to assess its future developments (Cheung et al. 2005). Horiuchi (2003) followed up the suggestion of Kannisto (2001) and argued that the modal age at death and the age distribution of death are probably the best indicators for studying a population's longevity. Indeed, the applications of this approach to the most recent data from some developed countries provide some useful information that may fuel the ongoing debate on the limits of human longevity, suggesting that those countries experiencing an increase in the modal age at death may be moving from a phase of 'compression of mortality' to a phase of 'shifting mortality'.

The continuous improvements of survival conditions explain, at least partly, why the last few decades have seen the notable increase in the number of centenarians, in particular 'super-centenarians' (110+) and 'semi-super-centenarians' (105-109) (Robine and Caselli 2005). Some scholars are trying to monitor these individuals, using various methodologies to count them and, above all, to confirm that the age indicated is actually true. Age validation is still the most important prerequisite for any study of centenarians (Perls et al. 1999; Allard and Robine 2000). Its absence is likely to bias the calculation of centenarian rates and, consequently, any explanation of this extreme longevity.

According to Perls et al. (2000), extreme longevity in a centenarian's family history supports the view of the inheritability of oldest-old longevity. This would imply an increased homogeneity among the oldest-old in connection with shared genetic factors (Vaupel et al. 1979; Vaupel et al. 1998). Indeed, recent findings indicate that increased homozygosity at loci involved in human longevity occurs, suggesting that two copies of the same allele may favour survival at later ages (Bonafé et al. 2003). This phenomenon is probably more frequent in consanguineous subjects-as in Sardinia—assuming that such alleles are recessive. Thus, longevity is present in many generations of the same families in spite of the great variations in life style and life expectancy during the last century. Moreover, the most recent studies on twins strongly support the view that genetic effects are important for survival at older ages, and that genetic influences on lifespan are minimal prior to age 60 (Hjelmborg et al. 2006).

Nevertheless, not all familial effects among extremely long-lived individuals are caused by genetic factors. Moreover, an exceptional number of persons in the same family reaching extreme old ages does not necessarily imply that genetic 
factors are important for human longevity. Shared characteristics, such as life style and the environment, might also be involved (Herskind et al. 1996; Caselli et al. 2006b). Despite the extensive and increasing information on the relationships between genetics and longevity, there is broad consensus that genetics can only partially account for the phenomenon, probably not more than $25-30 \%$. This has been demonstrated in many studies (see Christensen et al. 2006; Salvioli et al. 2006), including investigations that find different impacts by genetic factors, for instance with regard to gender and specific geographical territories. Human longevity is extremely complex and strongly influenced by external factors (environmental, cultural and economic conditions, stochastics etc.). It is still unknown to what extent genetics contribute to the relationships between these factors and longevity. This lack of knowledge motivates many bio-demographic studies analysing the non-genetic determinants of longevity.

Demography can also help to provide deeper insights into the role that inheritability might play in longevity, even in the absence of genetic information. This requires specific data such as the reconstruction of family histories of the oldest-old from distinct geographical areas (Caselli et al. 2006b). Genealogical data allow us also to study the relationship between parental age at childbearing and the longevity of their children. Some scholars hypothesise that there is an optimum age for procreation, with positive effects on the longevity of the children (see Caselli et al., Jarry et al. and Gavrilov and Gavrilova in this issue).

This 2013 issue of the Vienna Yearbook of Population Research tackles several of these aspects of human longevity from the demographic perspective. Despite the continuous rise of life expectancy there are still large differences between populations and sub-populations. The reasons for these differences are still not fully understood. The remaining knowledge gaps are primarily due to the highly complex network of factors that are causing inequalities in length of life. Although many risk factors for mortality have been identified (e.g. Cohen and Lee 1979; Cohen 1991; Cutler et al. 2006), it is unclear how they are related and how these relationships vary under different environments and life conditions. The existing knowledge about this network of factors is like a big puzzle consisting of many differently-sized pieces, not all of which have been discovered yet, and we are still far from putting them together to an overall picture. This issue of the Vienna Yearbook of Population Research aims at adding a good amount of new pieces to this puzzle and connecting them with some others whose position inside the overall picture is not yet clear. The articles are grouped into four sections: 'methodological issues for studying longevity and mortality', 'longevity islands', 'longevity and mortality of specific risk groups' and 'survival to old and oldest-old age: familial and socio-demographic determinants'.

\section{Methodological issues for studying longevity and mortality}

We have seen above that demographic research about the limits and determinants of human longevity cannot be conducted without considering methodological 
issues. Therefore, the first part of the present volume deals with several specific methodological aspects. The first article by Elizabeth Wrigley-Field (Mortality deceleration is not informative of unobserved heterogeneity in open groups) provides an in-depth exploration of the dynamics of mortality deceleration in the context of population sub-groups that are determined by their health status and can be entered as well as exited. For this purpose the author develops a theoretically simple dynamic frailty model in order to highlight the complexity in deceleration patterns resulting from movement between groups. She shows that the order of deceleration between the healthy and the not-healthy sub-populations is almost unpredictable in a dynamic context.

Shiro Horiuchi and colleagues (Modal age at death: lifespan indicator in the era of longevity extension) provide an extensive summary of their long research activity on the characteristics of the modal age at death $(\mathrm{M})$ as an indicator of human longevity. Their paper includes demonstrations with empirical data as well as derivations of statistical properties. The overview is complemented by analysing sex differences and country differences in mortality on the basis of M. Last but not least, the authors demonstrate how to estimate $\mathrm{M}$ from empirical data and they provide a new $\mathrm{R}$ routine to implement this estimation procedure.

The third methodological contribution by Jon Anson (Surviving to be oldest-olddestiny or chance?) shows that the probability of reaching old age seems unrelated to the survival conditions in younger ages. Moreover, the author shows by means of simulations that the emergence of extraordinary high centenarian rates in specific populations could occur by chance rather than being the consequence of causal mechanisms related to longevity. The author concludes that -in order to broaden our knowledge about the keys of extreme longevity - a conceptual framework is needed that identifies the conditions which are conducive to both extraordinary long and short lives.

\section{Longevity islands}

A promising strategy in mortality research is the identification of populations with exceptional longevity, as it is interesting to study the reasons for their low mortality levels. Some studies try to determine geographical areas where an exceptional proportion of people reach highest ages, which are called 'longevity islands', 'hot spots' or 'Blue Zones' (BZ). The second section of the Vienna Yearbook of Population Research 2013 focuses on such areas of unusual longevity. In the first article, Michel Poulain and colleagues (The Blue Zones: areas of exceptional longevity around the world) describe the $\mathrm{BZ}$ concept and discuss different indexes to measure exceptional longevity. They summarise existing validations of four specific BZ: Ogliastra in Sardinia, Okinawa in Japan, the Nicoya peninsula in Costa Rica and the island of Ikaria in Greece. The authors provide some new evidence for defining these areas as $\mathrm{BZ}$ and they perform a comparative examination of specific characteristics in order to find commonalities. At the end, Poulain et al. come to the 
conclusion that the extreme longevity of these BZ populations might be the result of a balance between the benefits of the traditional lifestyles and those of modernity.

Luis Rosero-Bixby and colleagues (The Nicoya region of Costa Rica: a high longevity island for elderly males) provide further evidence that Nicoya is in fact a BZ, though only for men. The paper extends existing knowledge by quantifying the longevity advantage of Nicoyan males and by investigating biomarkers, diet consumption patterns and other health indicators as possible explanations. The study indicates that the exceptional longevity of Nicoyan men is caused primarily by their low cardiovascular mortality, and the authors offer several possible explanations for this specific survival advantage.

Sebastian Klüsener and Rembrandt D. Scholz (Regional hot spots of exceptional longevity in Germany) present for the first time a study on longevity islands in Germany. Moreover, this is the first study that looks at the distances between place of birth and place at death of long-lived individuals (semi-supercentenarians). The authors identify some German longevity islands, including Berlin and some areas in north-western Germany as well as Munich. The authors find that these hot spots are (almost) identical for place of birth and place at death. The dominance of northern Germany is surprising since nowadays the regions of southern Germany show the highest life expectancy levels. The authors hypothesise that early- and late-life contextual conditions are the main explanations for the emergence of these BZ and consider also the possibility of genetic influences.

\section{Longevity and mortality of specific risk groups}

A circumstance that hampers advances in mortality research is the fact that single risk factors for mortality cannot be studied in isolation because they typically affect humans and their living conditions simultaneously. For instance, it is almost impossible to estimate how much of the differences in life expectancy by education is indeed a consequence of duration, intensity and specialisation of the acquired knowledge, i.e. of the factual effect of education. Usually, people with different education levels differ also in other socio-economic characteristics, such as occupation, income and lifestyle. An isolated examination of these factors would require laboratory experiments, which cannot be done with humans for obvious reasons. This is why mortality researchers are looking for specific sub-populations in which some risk factors are not existent or which are characterised by a specific risk factor. Another research strategy is the investigation of specific mortality paradoxes with the aim to better understand the linkage between specific risk factors. The third section of the present volume contains examples for all of these approaches.

Richard G. Rogers and colleagues (Lifetime abstainers and mortality risk in the United States) investigate the variability in mortality among non-drinkers, separated by their motivation to abstain from alcohol. They find that the risk of dying is low for light drinkers and for those non-drinkers who abstain from drinking for religious and moral reasons, have a responsibility to their family, were brought up not to drink 
and are not social. On the other side, mortality is higher among former, infrequent and moderate drinkers as well as some other groups of abstainers. Thus, the authors challenge the common picture of the J-shape relation between alcohol consumption and mortality and offer some explanations for this new perspective.

The study of Maria Winkler-Dworak and Heiner Kaden (The longevity of academicians: evidence from the Saxonian Academy of Sciences and Humanities in Leipzig) focuses on an interesting vanguard group in the achievement of longevity: the members of a learned society, here in particular the Saxonian Academy of Sciences and Humanities in Leipzig. They find that Saxonian academicians show a higher life expectancy than the total German male population and that the gap has widened since the 1950s. The results for the Saxonian academicians are similar to those of various other European learned societies. The authors conclude therefore that the survival of academicians is less determined by national mortality conditions but that they rather share a common health advantage that operates independently of economic conditions and societal environments.

Morgan E. Levine and Eileen M. Crimmins (Evidence of resiliency among longlived smokers) investigate the population subgroup of smokers aged 80 and older with a special focus on the effect of innate frailty. They find that smoking did not significantly contribute to the risk of mortality among individuals aged 80 and over, compared to smokers below age 80 who had higher mortality than non-smokers. Hence, long-lived smokers present an interesting paradox. They appear to present a distinct and biologically advantaged group, who are less susceptible to the negative effects of smoking and perhaps other environmental insults. Thus, the results of this study point at the practical importance of selection effects when differences in mortality are studied.

The study of Ethan J. Sharygin and Michel Guillot (Ethnicity, russification and excess mortality in Kazakhstan) investigates mortality differences between Russians and Kazakhs in Kazakhstan with the particular aim to test the 'Russian mortality paradox', that is that Russians in Central Asian countries are wealthier and better educated than the indigenous population but show worse health and higher mortality. The authors compare their results to those of a similar study for Kyrgyzstan, hypothesising that the ethnic differences are smaller in Kazakhstan and that overall adult mortality in Kazakhstan should lie between the levels apparent in Russia and Kyrgyzstan. The hypotheses are confirmed and the authors conclude that these results suggest the existence of a continuum between 'non-Russian' and 'Russian' mortality patterns, predominantly related to the extent of alcohol consumption.

\section{Survival to old and oldest-old age: familial and socio-demographic determinants}

The final section of the Vienna Yearbook of Population Research 2013 includes papers about the determinants of survival to old and oldest-old ages. The special 
focus of these articles is the extent to which extreme longevity is inherited or due to specific contextual and socio-demographic factors. Since Kirkwood and Rose (1991) suggested the existence of a trade-off between longevity and fertility, the relationship between these two phenomena has been tackled in various articles. The main results showed that women who live longer seem, on average, to have had fewer children and given birth to them at an apparently older age, particularly regarding their last children. Reviews of the existing literature can be found in the articles of this section.

The first paper by Graziella Caselli and colleagues (Exploring Sardinian longevity: women fertility and parental transmission of longevity) refers to the sample of the Sardinian centenarian study. The aims of this article are to analyse the twoway association between women fertility and longevity, and parental transmission of longevity. For the association between longevity and fertility, the authors find evidence that long-lived individuals benefit from factors related to the timing of fertility. Parental transmission of longevity seems to be significant for women, but not for men. The authors find further that a higher birth order decreases the probability of becoming a centenarian. Finally, they find a negative effect of a higher father age at birth on the longevity of female offspring. The authors conclude that long-lived women seem to have been favoured both by their late fertility and young paternal age at the moment of their own procreation.

The paper of Valérie Jarry et al. (Maternal age, birth order and other early life factors: a family-level approach to exploring exceptional survival) investigates very specific factors of early-life conditions and their effects on extreme longevity in Quebec. The core questions are why siblings are different from one another in terms of achieving exceptional longevity and if differences in parental age, birth order and other unshared variables could be associated with reaching old age. The most important findings are that having a younger mother is associated with a higher probability to become centenarian and that third and fourth-born individuals show a significant survival advantage. The authors conclude that the biological advantage of having a young mother is likely to be masked by a social disadvantage.

The paper of Leonid Gavrilov and Natalia Gavrilova (Determinants of exceptional human longevity: new ideas and findings) summarises the results of the authors' previous studies on centenarians, using several different approaches for specifying control groups of centenarians. In these studies, the authors identified several mechanisms that increase the probability to become a centenarian, including region of childhood residence, household property, genetics (parents' and siblings' longevity), lifestyles, maternal age at birth and month of birth. The authors suggest that studies on centenarians might prove to be a 'gold mine' in the search for the secrets of human longevity.

The last paper of Luisa Salaris and colleagues (Familial transmission of human longevity: a population-based study in an inland village of Sardinia (Italy), 1850 2010 ) is based on data collected by the authors on the population of a specific village in Sardinia for more than 150 years to examine the relationship between individuals' survival with that of their parents and siblings. They restrict the analysis to ages 50+ to exclude infant and child mortality as well as externally caused deaths (accidents 
etc.). The authors find effects of familial transmission of longevity, above all along the mother-daughter line and even more important between siblings, here especially for men.

In sum, the papers presented in this volume are very heterogeneous and they provide many new insights into the mechanisms behind extreme and unusual longevity. However, it is important to note that a very different issue than the length of human life is its quality in terms of health and well-being. Passing nowadays the threshold of 80 or 90 years opens up new horizons for remaining life expectancy. But if one's greatest desire is to live as long as possible, the aspiration is always to spend the additional life years in good health. The progress made in recent years in the fight against disease has been remarkable, and there is reasonable hope that even more effective treatments will become available in the near future. However, these aspects exceed the scope of the 2013 issue of the Vienna Yearbook of Population Research. A follow-up volume is planned that extends the presented findings about the quantity of human life by its qualitative characteristics, focusing on the analysis of trends and determinants of healthy life expectancy.

\section{Acknowledgements}

The 2013 issue of the Vienna Yearbook of Population Research is based on the contributions of the conference 'Determinants of Unusual and Differential Longevity', hosted by the Wittgenstein Centre for Demography and Global Human Capital (IIASA, VID/ÖAW, WU) and held in Vienna on 21-23 November 2012. The organisers of this conference-Marc Luy, Graziella Caselli, William P. Butz-are grateful to the participants and colleagues who helped in the organisation of the event as well as with the production of this journal issue, in particular Sylvia Trnka, Barbara Maschat, Angela Wiedemann, Werner Richter and Frank Kolesnik. We also thank the city of Vienna for their financial support of the conference and the referees who reviewed the submitted articles and helped the authors to improve their papers. Marc Luy received support from the European Research Council (ERC Starting Grant agreement No. 262663).

\section{References}

Allard, M., and J. M. Robine. 2000. Les Centenaires Francais. Etude De La Fondation IPSEN. Paris: Serdi Editores.

Barbi, E., G. Caselli, and J. Vallin. 2003. "Trajectories of Extreme Survival in Heterogeneous Populations". Population (English Edition) 58 (1): 43-66.

Bonafé, M., M. Barbieri, F. Marchegiani, F. Olivieri, E. Ragno, C. Giampieri, E. Mugianesi, M. Centurelli, C. Franceschi, and G. Paolisso. 2003. "Polymorphic Variants of Insulin-Like Growth Factor I (IGF-I) Receptor and Phosphoinositide 
3-Kinase Genes Affect IGF-I Plasma Levels and Human Longevity: Cues for an Evolutionarily Conserved Mechanism of Life Span Control". The Journal of Clinical Endocrinology $\mathcal{E}$ Metabolism 88 (7): 3299-3304. doi:10 . 1210 / jc . 2002-021810.

Capri, M., S. Salvioli, D. Monti, C. Caruso, G. Candore, S. Vasto, F. Olivieri, F. Marchegiani, P. Sansoni, G. Baggio, D. Mari, G. Passarino, G. De Benedictis, and C. Franceschi. 2008. "Human Longevity Within an Evolutionary Perspective: The Peculiar Paradigm of a Post-Reproductive Genetics". Experimental Gerontology 43 (2): 53-60. doi:10.1016/j . exger . 2007.06.004.

Caselli, G., L. Pozzi, J. W. Vaupel, L. Deiana, G. Pes, C. Carru, C. Franceschi, and G. Baggio. 2006a. "Family Clustering in Sardinian Longevity: A Genealogical Approach". Experimental Gerontology 41 (8): 727-736. doi:10 . 1016/j . exger . 2006.05 .009 .

Caselli, G., J. W. Vaupel, and A. I. Yashin. 2006b. "Explanation of the Decline in Mortality Among the Oldest-Old: A Demographic Point of View". In Human Longevity, Individual Life Duration, and the Growth of the Oldest-Old Population, ed. by J.-M. Robine, E. M. Crimmins, S. Horiuchi, and Z. Yi, 395-413. Dordrecht, The Netherlands: Springer.

Cheung, S. L. K., J.-M. Robine, E. J.-C. Tu, and G. Caselli. 2005. "Three Dimensions of the Survival Curve: Horizontalization, Verticalization, and Longevity Extension". Demography 42 (2): 243-258. doi:10.1353/dem. 2005. Q012.

Christensen, K., T. E. Johnson, and J. W. Vaupel. 2006. "The Quest for Genetic Determinants of Human Longevity: Challenges and Insights". Nature Reviews Genetics 7 (6): 436-448. doi:10.1038/nrg1871.

Cohen, B. L. 1991. "Catalog of Risks Extended and Updated". Health Physics 61 (3): 317-335.

Cohen, B. L., and I.-S. Lee. 1979. "A Catalog of Risks". Health Physics 36 (6): 707-722.

Comfort, A. 1956. The Biology of Senescence. New York, NY: Rinehart.

Comfort, A. 1964. Ageing. The Biology of Senescence. London: Routledge \& Kegan Paul.

Cutler, D. M., A. S. Deaton, and A. Lleras-Muney. 2006. "The Determinants of Mortality". Journal of Economic Perspectives 20 (3): 97-120.

Deevey, E. S. 1947. "Life Tables for Natural Populations of Animals". Journal of Vertebrate Paleontology 22: 283-314.

Finch, C. E., and M. C. Pike. 1996. "Maximum Life Span Predictions From the Gompertz Mortality Model". The Journals of Gerontology Series A: Biological Sciences and Medical Sciences 51 (3): B183-B194. 
Finch, C. E., and T. B. L. Kirkwood. 2000. Chance, Development, and Aging. Oxford: Oxford University Press.

Franceschi, C., D. Monti, D. Barbieri, E. Grassilli, L. Troiano, S. Salvioli, P. Negro, M. Capri, M. Guido, R. Azzi, R. Sansoni, R. Paganelli, U. Fagiolo, G. Baggio, S. Donazzan, S. Mariotti, S. D'addato, A. Gaddi, C. Ortolani, and A. Cossarizza. 1995. "Immunosenescence in Humans: Deterioration or Remodelling?" International Reviews of Immunology 12 (1): 57-74.

Franceschi, C., F. Olivieri, F. Marchegiani, M. Cardelli, L. Cavallone, M. Capri, S. Salvioli, S. Valensin, G. De Benedictis, A. Di Iorio, C. Caruso, G. Paolisso, and D. Monti. 2005. "Genes Involved in Immune Response/inflammation, IGF1/insulin Pathway and Response to Oxidative Stress Play a Major Role in the Genetics of Human Longevity: The Lesson of Centenarians". Mechanisms of Ageing and Development 126 (2): 351-361.

Fries, J. F. 1980. "Aging, Natural Death, and the Compression of Morbidity”. The New England Journal of Medicine 303 (3): 130-135.

Fries, J. F. 1984. "The Compression of Morbidity: Miscellaneous Comments About a Theme". The Gerontologist 24 (4): 354-359.

Gompertz, B. 1825. "On the Nature of the Function Expressive of the Law of Human Mortality, and on a New Mode of Determining the Value of Life Contingencies". Philosophical Transactions of the Royal Society of London 115: 513-583.

Hayflick, L. 1981. "Prospects for Human Life Extension by Genetic Manipulation". In Aging: A Challenge to Science and Society, ed. by D. D., N. W. Shock, and M. Marois, 1:162-179. New York: Oxford University Press.

Herskind, A. M., M. McGue, N. V. Holm, T. I. A. Sørensen, B. Harvald, and J. W. Vaupel. 1996. "The Heritability of Human Longevity: A Population-Based Study of 2872 Danish Twin Pairs Born 1870-1900". Human Genetics 97 (3): 319-323. doi:10.1007/BF02185763.

Hjelmborg, J. v., I. Iachine, A. Skytthe, J. W. Vaupel, M. McGue, M. Koskenvuo, J. Kaprio, N. L. Pedersen, and K. Christensen. 2006. "Genetic Influence on Human Lifespan and Longevity". Human Genetics 119 (3): 312-321. doi:10 . 1007 / s00439-006-0144-y.

Horiuchi, S. 2003. "Interspecies Differences in the Life Span Distribution: Humans Versus Invertebrates". In Life Span: Evolutionary, Ecological, and Demographic Perspective, ed. by J. R. Carey and S. Tuljapurkar, 127-151. Supplement to Population and Development Review 29. New York: Population Council.

Kannisto, V. 2001. "Mode and Dispersion of the Length of Life". Population (English Edition) 13 (1): 159-171.

Kirkwood, T. B. L. 1990. “The Disposable Soma Theory of Aging”. In Genetic Effects on Aging II, ed. by D. Harrison, 9-19. Caldwell, NJ: Telford.

Kirkwood, T. B. L., and C. Franceschi. 1992. "Is Aging as Complex as It Would Appear?" Annals of the New York Academy of Sciences 663: 412-417. 
Kirkwood, T. B. L., and M. R. Rose. 1991. "Evolution of Senescence: Late Survival Sacrificed for Reproduction". Philosophical Transactions of the Royal Society of London. Series B: Biological Sciences 332 (1262): 15-24. doi:10. 1098/rstb . 1991.0028.

Medvedev, Z. A. 1990. "An Attempt at a Rational Classification of Theories of Ageing”. Biological Reviews 65 (3): 375-398.

Oeppen, J., and J. W. Vaupel. 2002. "Broken Limits to Life Expectancy". Science 296 (5570): 1029-1031.

Olshansky, S. J., B. A. Carnes, and A. Désesquelles. 2001. "Demography: Prospects for Human Longevity”. Science 291 (5508): 1491-1492.

Pearl, R., and C. R. Doering. 1923. "A Comparison of the Mortality of Certain Lower Organisms With That of Man”. Science 57 (1468): 209-212.

Perls, T. T., K. Bochen, M. Freeman, L. Alpert, and M. H. Silver. 1999. "Validity of Reported Age and Centenarian Prevalence in New England". Age and Ageing 28 (2): 193-197.

Pes, G. M., D. Lio, C. Carru, L. Deiana, G. Baggio, C. Franceschi, L. Ferrucci, F. Oliveri, L. Scola, A. Crivello, G. Candore, G. Colonna-Romano, and C. Caruso. 2004. "Association Between Longevity and Cytokine Gene Polymorphisms: A Study in Sardinian Centenarians". Aging Clinical and Experimental Research 16 (3): 244-248.

Robine, J.-M., and G. Caselli. 2005. “An Unprecedented Increase in the Number of Centenarians”. Genus 61 (1): 57-82.

Robine, J.-M., G. Caselli, D. Rasulo, and A. Cournil. 2006a. "Differentials in the Femininity Ratio Among Centenarians: Variations Between Northern and Southern Italy From 1870”. Population Studies 66 (1): 99-113.

Robine, J.-M., E. M. Crimmins, Y. Zeng, and S. Horiuchi. 2006b. Human Longevity, Individual Life Duration, and the Growth of the Oldest-Old Population. International Studies in Population. Dordrecht, The Netherlands: Springer.

Salvioli, S., F. Olivieri, F. Marchegiani, M. Cardelli, A. Santoro, E. Bellavista, M. Mishto, L. Invidia, M. Capri, S. Valensin, F. Sevini, E. Cevenini, L. Celani, F. Lescai, E. Gonos, C. Caruso, G. Paolisso, G. De Benedictis, D. Monti, and C. Franceschi. 2006. "Genes, Ageing and Longevity in Humans: Problems, Advantages and Perspectives". Free Radical Research 40 (12): 1303-1323.

Vaupel, J. W., J. R. Carey, K. Christensen, T. E. Johnson, A. I. Yashin, N. V. Holm, I. A. Iachine, V. Kannisto, A. A. Khazaeli, P. Liedo, V. D. Longo, Y. Zeng, K. G. Manton, and J. W. Curtsinger. 1998. "Biodemographic Trajectories of Longevity”. Science 280 (5365): 855-860. doi:10. 1126/science. 280. 5365 . 855.

Vaupel, J. W., K. G. Manton, and E. Stallard. 1979. "The Impact of Heterogeneity in Individual Frailty on the Dynamics of Mortality". Demography 16 (3): 439-454. 
Williams, G. C. 2001. "Pleiotropy, Natural Selection, and the Evolution of Senescence". Evolution 11 (4): 13.

Wilmoth, J. R. 2001. "How Long Can We Live? A Review Essay". Population and Development Review 27 (4): 791-809.

Wilmoth, J. R., L. J. Deegan, H. Lundström, and S. Horiuchi. 2000. "Increase of Maximum Life-Span in Sweden, 1861-1999”. Science 289 (5488): 2366-2368. doi:10.1126/science.289.5488.2366.

Wilmoth, J. R., and S. Horiuchi. 1999. "Rectangularization Revisited: Variability of Age at Death Within Human Populations". Demography 36 (4): 475-495. 